\title{
Capacidad Funcional en Adultos Mayores que Asisten a un Programa de Rehabilitación Cardiaca
}

\section{Functional Ability in Adults who Take Part in a Cardiovascular Disease Rehabilitation Program}

Recibido: 15 de Nov. 2013/Enviado para modificación: 19 de Ene. 2014/Aceptado: 10 de Feb. 2014

\author{
Narledis Núñez Bravo ${ }^{1 *}$ \\ Universidad Simón Bolívar
}

\section{RESUMEN}

Introducción: En la edad adulta, se experimentan cambios estructurales y funcionales, los adultos mayores que presentan alguna enfermedad, especialmente si es crónica, como es el caso de las enfermedades cardiovasculares, tiene mayor probabilidad de presentar alteraciones en su funcionamiento. Objetivo: Caracterizar la capacidad funcional en adultos mayores con enfermedad cardiovascular, que asisten a un programa de rehabilitación cardiovascular en una clínica privada del distrito de Barranquilla. Materiales y métodos: Se realizó un estudio descriptivo en 18 adultos mayores entre 60 y 89 años de edad. Utilizando la escala de Barthel para evaluar las actividades de la vida diaria, la escala de Lawton, para las actividades instrumentales de la vida diaria. Y por medio de una encuesta estructurada, se identificó la participación en actividades sociales y recreativas. Resultados: El $60 \%$ de los sujetos que se encontraban en la quinta semana de tratamiento presentaron dependencia leve en la realización de las Actividades Básicas de la Vida Diaria; mientras que el 100\% de los adultos de género masculino que se hallaban en la quinta y octava semana de tratamiento presentaron ligera dependencia en las Actividades Instrumentales de la Vida Diaria. Las actividades en las que necesitan mayor ayuda son las relacionadas con el manejo de asuntos económicos, en particular los sujetos que se encuentran en la octava semana de tratamiento. Conclusión: Los cuidadores y/o familiares influyen en el desarrollo de las actividades, lo cual puede deberse al temor de que el esfuerzo genere complicaciones en el estado actual de la persona mayor.

Palabras Clave: Adulto mayor, actividades cotidianas, enfermedades cardiovasculares, autonomía personal (Fuente: DeCS).

\section{ABSTRACT}

Introduction: In the adult age, there are experienced structural and functional changes, the major adults who present some disease, especially if it is chronic, since it is the case of the cardiovascular diseases; it has major probability of presenting alterations in his functioning. Objective: To characterize the functional ability in adults with cardiovascular disease who take part in a cardiovascular rehabilitation program in a private clinic in Barranquilla. Materials and Methods: 18 adults between 60 and 89 years of age were sampled in a descriptive study. In order to evaluate activities of daily living, Barthel Scale was applied to assess instrumental activities of daily living Lawton Scale was used and through a well structured survey the participation in social and recreational activities was identified. Results: $60 \%$ of the subjects who are in the fifth week of treatment shows mild dependency in the achievement of the basic activities of daily living was demonstrated (ABVD). $100 \%$ of male adults who are in the fifth and eighth week of treatment show mild dependency in relation with instrumental activities of daily living (AIVD). In the activities in which subjects who are in the eighth week of treatment need help mainly is in economic matters handling. Social and recreational activities are shown in familiar meetings whereas $100 \%$ of the subjects do not do sports activities. Conclusion: The caregivers and/or relatives influence dependency in relation with the development of the activities, which can be based on the fear that the subject effort generates complications of the current state of the person.

Keywords: aged, activities of daily living, cardiovascular diseases, personal autonomy (Source: MeSH, NLM).

Para citar este artículo: Núñez BN. Capacidad funcional en adultos mayores que asisten a un programa de rehabilitación cardiaca. Cienc. innov. salud. 2014; 2 (1): 33-39.

1 Fisioterapeuta. Est. Maestría en Desarrollo y Gestión de Empresas Sociales. Docente investigadora Programa de Fisioterapia. Miembro del grupo Familia y Desarrollo Humano. Universidad Simón Bolívar. Correo electrónico: nnunez3@ unisimonbolivar.edu.co

* Investigación realizada durante la beca-pasantía como joven investigador e innovador "Virginia Gutiérrez de Pineda", convocatoria 510 del 2010 de Colciencias. 


\section{Introducción}

El envejecimiento de la población mundial es un fenómeno progresivo. A partir de 1950, la proporción de adultos mayores viene aumentando regularmente. Así, de un $8 \%$ reportado en 1950 , pasó al $11 \%$ en 2009, y se calcula que en 2050 llegará al 22\%, siempre y cuando la mortalidad siga disminuyendo en las personas mayores y la fecundidad tienda a bajar (1).

En el año 2000, había en el mundo 600 millones de personas de 60 años o más, dato que triplicó la cifra presentada en 1950, y que para el año 2009 había sobrepasado los 700 millones. Esto permite proyectar que para el año 2050, habrá 2000 millones de adultos mayores, lo que muestra que este grupo de edad se habrá triplicado en un lapso de 50 años. A nivel mundial, la población de personas mayores aumenta a razón del 2,6\% por año, superando en mucho la población total, que aumenta a razón de un $1,2 \%$ por año. De este modo, se calcula que, al menos hasta 2050, la población de adultos mayores seguirá aumentando a gran escala en relación con el resto de la población (1).

Mirando otro aspecto del problema, se halla que, según la Comisión Económica para América Latina y el Caribe (CEPAL), los países se pueden clasificar de acuerdo con la etapa en que se encuentren en su proceso de envejecimiento (2). Colombia se ubica en el segundo grupo, denominado envejecimiento moderado, con proporciones entre el $6 \%$ y el $8 \%$, que para el 2050 sobrepasarían el 20\% (2).

Se debe considerar que el envejecimiento es un proceso natural, universal, que ocurre en todos los seres vivos y se caracteriza por ser irreversible, progresivo, heterogéneo, deletéreo y multifactorial. Tiene lugar durante la última etapa del ciclo vital, presentándose como una disminución progresiva de la reserva en la capacidad funcional $y$, en consecuencia, en una menor habilidad de adaptación a factores del medio ambiente (3). Por lo tanto, se puede decir que es un proceso biológico, social y psicológico, como resultado de la interacción de la herencia, el ambiente y la conducta.

También se puede entender la vejez como el conjunto de cambios biológicos, psicológicos y sociales, normales e inherentes a todo individuo, que deja huella en el aspecto físico y en el comportamiento de cada uno (4).
De manera concreta, en la edad adulta, se experimenta el deterioro de las funciones orgánicas, siendo este un fenómeno irreversible que incluye cambios estructurales y funcionales (5). Por ello, a medida que las personas envejecen, aumenta la probabilidad de presentar limitaciones funcionales, y así, el adulto mayor que presenta alguna enfermedad, especialmente si es crónica, como es el caso de las dolencias cardiovasculares, tiene mayor probabilidad de presentar alteraciones en su funcionamiento, tanto mental como físico (4).

Según la Organización Mundial de la Salud (OMS), la enfermedad cardiovascular es uno de los mayores problemas de salud pública en el mundo, además de ser la primera causa de mortalidad, al ocasionar 17 millones de muertes al año (6). La enfermedad cardiovascular es responsable de 32 millones de síndromes coronarios y accidentes cerebro-vasculares, de los cuales, entre el 40-70 \% son fatales en países desarrollados (6). Se estima que este problema es mucho mayor en países en vía de desarrollo, y de hecho, en Colombia constituye la segunda causa de muerte (7).

Un evento cardiovascular puede reducir o alterar la capacidad funcional de las personas $\mathrm{y}$, por ende, afectar el desempeño adecuado en el ámbito físico, familiar, social y laboral (8). La misma OMS ha definido la salud de los adultos mayores por su capacidad para funcionar y no por sus limitaciones, discapacidades o enfermedades que presente (9). Así, la calidad de vida de los adultos mayores está estrechamente relacionada con la capacidad funcional y el conjunto de condiciones que le permiten participar en su propio autocuidado, en la vida familiar y social (9).

En este orden de ideas, la capacidad funcional se define como la facultad de ejecutar tareas y desempeñar roles sociales en la cotidianidad, dentro de un amplio rango de complejidad, con funcionalidad, independencia y autonomía. A su vez, la funcionalidad o independencia funcional es la capacidad de cumplir acciones requeridas en el diario vivir, para mantener el cuerpo y subsistir independientemente (10). Cuando el cuerpo y la mente son capaces de llevar a cabo estas actividades de la vida cotidiana se dice que la funcionalidad esta indemne (10).

En este sentido, las Actividades de la Vida Diaria (AVD) son elementos claves para medir la calidad de

Cienc. innov. salud. Junio 2014; 2 (1):33 - 39. Universidad Simón Bolívar (Col). ISSN: 2344-8636 http://portal.unisimonbolivar.edu.co:82/rdigital/innovacionsalud 
vida y el estatus funcional en los adultos mayores. Estas abarcan las Actividades Básicas de la Vida Diaria (ABVD), que se refieren al mantenimiento personal en términos de supervivencia física; Actividades Instrumentales de la Vida Diaria (AIVD), que comprenden el mantenimiento personal en términos de supervivencia cultural; y las actividades de ocio y actividades sociales, que aluden a actividades comunitarias y auto-enriquecedoras (11).

Existen situaciones naturales que pueden crear barreras a los adultos mayores para la ejecución de las actividades de la vida diaria, así como situaciones sociales. Entre todas las primeras se cuentan: la alta prevalencia de las enfermedades crónicas, secuelas propias de estas y cambios físicos que acompañan el proceso de envejecimiento (12). Según la población envejece, aumenta la prevalencia de enfermedades crónicas y limitaciones en las actividades. Las alteraciones cardiovasculares que comprometen la capacidad funcional destacan entre las patologías comunes en adultos mayores, las cuales causan aproximadamente una cuarta parte de todas las muertes en el mundo (13).

El tratamiento de los pacientes con enfermedades cardiovasculares suele ser manejado por un equipo interdisciplinario que busca reducir el impacto físico y psicosocial de las condiciones limitantes del individuo, además de aumentar la capacidad funcional, y de mejorar la calidad de vida y el pronóstico del paciente (14). Precisamente, a partir de los anteriores planteamientos, esta investigación se planteó el objetivo de caracterizar la capacidad funcional en adultos mayores con enfermedad cardiovascular, que asisten a un programa de rehabilitación cardiovascular fase III en una clínica privada de la ciudad de Barranquilla (Colombia).

\section{Materiales y Métodos}

Como se acaba de expresar, este artículo es resultado de un estudio descriptivo realizado en adultos mayores diagnosticados con enfermedad cardiovascular, que asisten al programa de rehabilitación cardiovascular de una clínica privada de Barranquilla. La población total estuvo conformada por 60 personas, pero se escogió una muestra de 18 personas que cumplieron con los siguientes criterios: ser mayores de 60 años, hallarse en tratamiento de rehabilitación cardiovascular fase III y que aceptaron participar voluntariamente en el estudio. Los sujetos se encontraban en diferentes semanas de tratamiento, así: 5 sujetos se encontraban en la quinta semana, 1 sujeto en la octava semana, 5 adultos mayores se hallaban en la 15 semana y 7 sujetos llevaban entre 26 y más semanas de intervención.

Previamente, los pacientes firmaron el consentimiento informado. Luego, mediante la escala de Barthel, se evaluaron las AVD; en tanto que la escala de Lawton se utilizó para establecer la autonomía de los sujetos de estudio durante las AIVD. También, se aplicó una encuesta estructurada para las actividades sociales y recreativas.

La escala de Barthel es utilizada para evaluar la independencia funcional de las personas, a través de 10 tipos de actividades: alimentación, bañarse, arreglarse, vestirse, micción, deposición, uso de sanitario, trasladarse sillón/cama, deambulación y subir escaleras. Esta escala categoriza a los sujetos según la siguiente puntuación: 100 independencia, $>60$ dependiente leve, 55/40 dependiente moderado, 35/20 dependiente severo, <20 dependiente total (13).

La escala de Lawton comprende 8 ítems, que se refieren a la capacidad para: utilizar el teléfono, hacer compras, preparación de la comida, cuidado de la casa, lavado de la ropa, uso de medios de transporte, responsabilidad respecto a la medicación y administración de su economía y define, por último, si una persona es independiente o dependiente. La máxima dependencia estaría marcada por la obtención de 0 puntos, mientras que una suma de 8 puntos expresaría una independencia total. Esta escala categoriza el grado de dependencia de acuerdo con los resultados, así: en hombres, sin evaluar las actividades de preparación de comida, lavado de ropa y cuidado de la casa, 0 como dependencia total, 1 severa, 2 o 3 moderada, 4 ligera, 5 autónomo. En mujeres, la puntuación se valora de otro modo: 0-1 indica dependencia total; 2 o 3 , dependencia severa; 4 o 5 , dependencia moderada; 6 o 7, ligera dependencia; y 8, autonomía (15).

Finalmente, la encuesta estructurada sobre la realización de actividades sociales y recreativas, se utilizó para identificar el nivel de participación de los adultos mayores en juegos de mesa, baile, manualidades, ejercicios dirigidos, ejercicios libres, deporte, caminatas, escuchar radio, leer periódico, leer libros, ver televisión y asistir a reuniones familiares.

Cienc. innov. salud. Junio 2014; 2 (1):33 - 39. Universidad Simón Bolívar (Col). ISSN: 2344-8636 
El análisis estadístico se realizó mediante el paquete SPSS versión 18.0, y los resultados se presentan en frecuencias absolutas y relativas.

\section{Resultados}

De los sujetos participantes, el 61,1\% tenía entre 60 a 69 años de edad. Con una media de 68,8 años. El $55,5 \%$ de la muestra era de género masculino y el $44,4 \%$, de género femenino. El estado civil con mayor porcentaje es casado con un 50\%; seguido por viudo en un $33.3 \%$. En cuanto al nivel de escolaridad, se encontró que el 44,4\% de los adultos mayores sólo cuentan con el nivel primario, seguido por el nivel técnico en un $22,2 \%$ (Tabla 1 ).

Tabla 1. Características generales de la población participante

\begin{tabular}{llcc}
\hline VARIABLE & Niveles & Frec. Obs. & Frec. \% \\
\hline \multirow{3}{*}{ Rango etáreo } & $60-69$ & 11 & $61,10 \%$ \\
& $70-79$ & 4 & $22,20 \%$ \\
& $80-89$ & 3 & $16,60 \%$ \\
\hline \multirow{2}{*}{ Género } & Mujeres & 8 & $44,40 \%$ \\
& Hombres & 10 & $55,50 \%$ \\
\hline \multirow{4}{*}{ Estado civil } & Casado & 9 & $50 \%$ \\
& Unión libre & 2 & $11,10 \%$ \\
& Divorciado & 1 & $5,60 \%$ \\
& Viudo & 6 & $33,30 \%$ \\
\hline \multirow{3}{*}{ Nivel de } & Primaria & 8 & $44,40 \%$ \\
escolaridad & Secundaria & 3 & $16,70 \%$ \\
& Técnico & 4 & $22,20 \%$ \\
& Profesional & 3 & $16,70 \%$ \\
\hline
\end{tabular}

En la Tabla 2, se observa, por otra parte, que los sujetos que se encontraban en la quinta semana de tratamiento presentaban el mayor porcentaje de dependencia, con un $60 \%$ de dependencia leve. Las actividades que presentaron mayor dependencia fueron las de subir y bajar escaleras. Concretamente, en la semana 5 de tratamiento, el $40 \%$ refirió ser totalmente dependiente para realizar esta actividad y un $20 \%$ dijo necesitar ayuda.
Tabla 2. Valoración funcional de las ABVD

\begin{tabular}{llcccc}
\hline \multirow{2}{*}{ VARIABLES } & \multirow{2}{*}{ Niveles Variable } & \multicolumn{4}{c}{ Semanas de tratamiento } \\
\cline { 3 - 6 } & & $\mathbf{5 ~ s e m}$ & $\mathbf{8 ~ s e m}$ & $\mathbf{1 5}$ sem & $\geq 26$ sem \\
\hline \multirow{2}{*}{ Alimentación } & Independiente & $5(100 \%)$ & $1(100 \%)$ & $5(100 \%)$ & $7(100 \%)$ \\
& Dependiente & $0(0 \%)$ & $0(0 \%)$ & $0(0 \%)$ & $0(0 \%)$ \\
\hline \multirow{2}{*}{ Bañarse } & Independiente & $5(100 \%)$ & $1(100 \%)$ & $5(100 \%)$ & $7(100 \%)$ \\
& Dependiente & $0(0 \%)$ & $0(0 \%)$ & $0(0 \%)$ & $0(0 \%)$ \\
\hline \multirow{2}{*}{ Aseo personal } & Independiente & $5(100 \%)$ & $1(100 \%)$ & $5(100 \%)$ & $7(100 \%)$ \\
& Dependiente & $0(0 \%)$ & $0(0 \%)$ & $0(0 \%)$ & $0(0 \%)$ \\
\hline Vestirse y & Independiente & $5(100 \%)$ & $1(100 \%)$ & $5(100 \%)$ & $6(85,7 \%)$ \\
desvestirse & Necesita ayuda & $0(0 \%)$ & $0(0 \%)$ & $0(0 \%)$ & $1(14,3 \%)$ \\
\hline \multirow{2}{*}{ Micción } & Continente & $5(100 \%)$ & $1(100 \%)$ & $5(100 \%)$ & $7(100 \%)$ \\
& Incontinente & $0(0 \%)$ & $0(0 \%)$ & $0(0 \%)$ & $0(0 \%)$ \\
\hline \multirow{2}{*}{ Deposición } & Continente & $5(100 \%)$ & $1(100 \%)$ & $5(100 \%)$ & $7(100 \%)$ \\
& Incontinente & $0(0 \%)$ & $0(0 \%)$ & $0(0 \%)$ & $0(0 \%)$ \\
\hline \multirow{2}{*}{ Uso del } & Independiente & $5(100 \%)$ & $1(100 \%)$ & $5(100 \%)$ & $7(100 \%)$ \\
Sanitario & Dependiente & $0(0 \%)$ & $0(0 \%)$ & $0(0 \%)$ & $0(0 \%)$ \\
\hline \multirow{2}{*}{ Trasladarse } & Independiente & $5(100 \%)$ & $1(100 \%)$ & $5(100 \%)$ & $6(85,7 \%)$ \\
sillón-cama & Mínima ayuda & $0(0 \%)$ & $0(0 \%)$ & $0(0 \%)$ & $1(14,3 \%)$ \\
\hline \multirow{2}{*}{ Deambulación } & Independiente & $5(100 \%)$ & $1(100 \%)$ & $5(100 \%)$ & $7(100 \%)$ \\
& Dependiente & $0(0 \%)$ & $0(0 \%)$ & $0(0 \%)$ & $0(0 \%)$ \\
\hline \multirow{2}{*}{ Subir y bajar } & Independiente & $2(40 \%)$ & $1(100 \%)$ & $5(100 \%)$ & $6(85,7 \%)$ \\
escaleras & Necesita ayuda & $1(20 \%)$ & $0(0 \%)$ & $0(0 \%)$ & $0(0 \%)$ \\
& Dependiente & $2(40 \%)$ & $0(0 \%)$ & $0(0 \%)$ & $1(14,3 \%)$ \\
\hline Niveles de & Independencia & $2(40 \%)$ & $100 \%$ & $100 \%$ & $6(85,7 \%)$ \\
dependencia & Dependencia leve & $3(60 \%)$ & $0(0 \%)$ & $0 \%$ & $1(14,3 \%)$ \\
\hline & & & & & \\
& & & &
\end{tabular}

En relación con las AIVD, en los hombres, el 100\% de la población que se encontraba en la 5 semana de tratamiento presentó ligera dependencia, al igual que las personas que se hallaban en la 8 semana de tratamiento. El 50\% de los sujetos que se encontraban en la 15 semana presentaron ligera dependencia, y un $25 \%$ moderada dependencia. Los sujetos que llevaban $\geq 26$ semana manifestaron ser autónomos en un $75 \%$ (Tabla 3).

Tabla 3. Valoración funcional de las AIVD en los hombres

\begin{tabular}{|c|c|c|c|c|c|}
\hline \multirow{2}{*}{ Variables } & \multirow{2}{*}{ Niveles Variable } & \multicolumn{4}{|c|}{ Semanas de tratamiento } \\
\hline & & $5 \mathrm{sem}$ & $8 \mathrm{sem}$ & 15 sem & $\geq 26$ sem \\
\hline \multirow{2}{*}{$\begin{array}{l}\text { Capacidad } \\
\text { para usar el } \\
\text { teléfono }\end{array}$} & $\begin{array}{l}\text { Utiliza el teléfono por } \\
\text { iniciativa propia }\end{array}$ & $1(100 \%)$ & $1(100 \%)$ & $4(100 \%)$ & $4(100 \%)$ \\
\hline & $\begin{array}{l}\text { No es capaz de } \\
\text { utilizar el teléfono }\end{array}$ & $0(0 \%)$ & $0(0 \%)$ & $0(0 \%)$ & $0(0 \%)$ \\
\hline \multirow[b]{2}{*}{$\begin{array}{l}\text { Hacer } \\
\text { compras }\end{array}$} & $\begin{array}{l}\text { Totalmente incapaz } \\
\text { de comprar }\end{array}$ & $1(100 \%)$ & $0(0 \%)$ & $1(25 \%)$ & $1(25 \%)$ \\
\hline & $\begin{array}{l}\text { Realiza todas las } \\
\text { compras necesarias } \\
\text { independientemente }\end{array}$ & $0(0 \%)$ & $1(100 \%)$ & $3(75 \%)$ & $3(75 \%)$ \\
\hline \multirow[t]{2}{*}{$\begin{array}{l}\text { Uso de } \\
\text { medios de } \\
\text { transporte }\end{array}$} & $\begin{array}{l}\text { Viaja solo en } \\
\text { transporte público o } \\
\text { conduce su propio } \\
\text { coche }\end{array}$ & $1(100 \%)$ & $1(100 \%)$ & $4(100 \%)$ & $4(100 \%)$ \\
\hline & No viaja & $0(0 \%)$ & $0(0 \%)$ & $0(0 \%)$ & $0(0 \%)$ \\
\hline \multirow{2}{*}{$\begin{array}{l}\text { Responsabili } \\
\text { dad respecto } \\
\text { a su } \\
\text { medicación }\end{array}$} & $\begin{array}{l}\text { Toma su medicación } \\
\text { si la dosis le es } \\
\text { preparada } \\
\text { previamente }\end{array}$ & $0(0 \%)$ & $0(0 \%)$ & $1(25 \%)$ & $1(25 \%)$ \\
\hline & $\begin{array}{l}\text { Es capaz de tomar su } \\
\text { medicación a la hora } \\
\text { y con la dosis correcta }\end{array}$ & $1(100 \%)$ & $1(100 \%)$ & $3(75 \%)$ & $3(75 \%)$ \\
\hline \multirow{2}{*}{$\begin{array}{l}\text { Manejo de } \\
\text { asuntos } \\
\text { económicos }\end{array}$} & $\begin{array}{l}\text { Incapaz de manejar } \\
\text { dinero }\end{array}$ & $0(0 \%)$ & $1(100 \%)$ & $2(50 \%)$ & $1(25 \%)$ \\
\hline & $\begin{array}{l}\text { Se encarga de sus } \\
\text { asuntos económicos } \\
\text { por sí solo }\end{array}$ & $1(100 \%)$ & $0(0 \%)$ & $2(50 \%)$ & $3(75 \%)$ \\
\hline \multirow{3}{*}{$\begin{array}{l}\text { Nivel de } \\
\text { dependencia }\end{array}$} & Moderada & $0(0 \%)$ & $0(0 \%)$ & $1(25 \%)$ & $1(25 \%)$ \\
\hline & Ligera & $1(100 \%)$ & $1(100 \%)$ & $2(50 \%)$ & $0(0 \%)$ \\
\hline & Autónomo & $0(0 \%)$ & $0(0 \%)$ & $1(25 \%)$ & $3(75 \%)$ \\
\hline
\end{tabular}

Cienc. innov. salud. Junio 2014; 2 (1):33 - 39. Universidad Simón Bolívar (Col). ISSN: 2344-8636

http://portal.unisimonbolivar.edu.co:82/rdigital/innovacionsalud 
En las actividades que mayormente necesitan ayuda estos pacientes es en el manejo de asuntos económicos. El $100 \%$ de los sujetos que se encontraban en la semana 8 refirieron, en efecto, que son incapaces de manejar su dinero, mientras que el $50 \%$ de los sujetos que estaban en la 15 semana afirmaron que no pueden realizar esta actividad.

Tabla 4. Valoración funcional de las AIVD en las mujeres

\begin{tabular}{|c|c|c|c|c|c|}
\hline \multirow{2}{*}{ Variables } & \multirow{2}{*}{ Niveles Variable } & \multicolumn{4}{|c|}{ Semanas de tratamiento } \\
\hline & & 5 sem & $8 \mathrm{sem}$ & 15 sem & $\geq 26$ sem \\
\hline \multirow{2}{*}{$\begin{array}{l}\text { Capacidad } \\
\text { para usar el } \\
\text { teléfono }\end{array}$} & $\begin{array}{l}\text { Utiliza el } \\
\text { teléfono por } \\
\text { iniciativa propia }\end{array}$ & - & - & - & - \\
\hline & $\begin{array}{l}\text { No es capaz de } \\
\text { utilizar el } \\
\text { teléfono }\end{array}$ & $4(100 \%)$ & $1(100 \%)$ & $3(100 \%)$ & $4(100 \%)$ \\
\hline \multirow{3}{*}{$\begin{array}{l}\text { Hacer } \\
\text { compras }\end{array}$} & $\begin{array}{l}\text { Totalmente } \\
\text { incapaz de } \\
\text { comprar }\end{array}$ & $1(100 \%)$ & $0(0 \%)$ & $1(25 \%)$ & $1(25 \%)$ \\
\hline & $\begin{array}{l}\text { Realiza todas las } \\
\text { compras }\end{array}$ & & & & \\
\hline & $\begin{array}{l}\text { necesarias } \\
\text { independienteme } \\
\text { nte }\end{array}$ & $0(0 \%)$ & $1(100 \%)$ & $3(75 \%)$ & $3(75 \%)$ \\
\hline \multirow{2}{*}{$\begin{array}{l}\text { Uso de } \\
\text { medios de } \\
\text { transporte }\end{array}$} & $\begin{array}{l}\text { Viaja solo en } \\
\text { transporte } \\
\text { público o }\end{array}$ & $1(100 \%)$ & $1(100 \%)$ & $4(100 \%)$ & $4(100 \%)$ \\
\hline & $\begin{array}{l}\text { propio coche } \\
\text { No viaja }\end{array}$ & $0(0 \%)$ & $0(0 \%)$ & $0(0 \%)$ & $0(0 \%)$ \\
\hline \multirow{2}{*}{$\begin{array}{l}\text { Responsabili } \\
\text { dad respecto } \\
\text { a su } \\
\text { medicación }\end{array}$} & $\begin{array}{l}\text { Toma su } \\
\text { medicación si la } \\
\text { dosis le es } \\
\text { preparada } \\
\text { previamente }\end{array}$ & $0(0 \%)$ & $0(0 \%)$ & $1(25 \%)$ & $1(25 \%)$ \\
\hline & $\begin{array}{l}\text { Es capaz de } \\
\text { tomar su } \\
\text { medicación a la } \\
\text { hora y con la } \\
\text { dosis correcta }\end{array}$ & $1(100 \%)$ & $1(100 \%)$ & $3(75 \%)$ & $3(75 \%)$ \\
\hline \multirow[b]{2}{*}{$\begin{array}{l}\text { Manejo de } \\
\text { asuntos } \\
\text { económicos }\end{array}$} & $\begin{array}{l}\text { Incapaz de } \\
\text { manejar dinero }\end{array}$ & $0(0 \%)$ & $1(100 \%)$ & $2(50 \%)$ & $1(25 \%)$ \\
\hline & $\begin{array}{l}\text { Se encarga de sus } \\
\text { asuntos } \\
\text { económicos por } \\
\text { sí solo }\end{array}$ & $1(100 \%)$ & $0(0 \%)$ & $2(50 \%)$ & $3(75 \%)$ \\
\hline \multirow{3}{*}{$\begin{array}{l}\text { Nivel de } \\
\text { dependencia }\end{array}$} & Moderada & $0(0 \%)$ & $0(0 \%)$ & $1(25 \%)$ & $1(25 \%)$ \\
\hline & Ligera & $1(100 \%)$ & $1(100 \%)$ & $2(50 \%)$ & $0(0 \%)$ \\
\hline & Autónomo & $0(0 \%)$ & $0(0 \%)$ & $1(25 \%)$ & $3(75 \%)$ \\
\hline
\end{tabular}

Respecto a los adultos mayores de género femenino, en la Tabla 4 se observa que quienes se hallaban en la 5 semana presentaron $25 \%$ de dependencia total, severa, moderada y ligera. Las mujeres que llevaban 15 semanas de tratamiento presentaron un $100 \%$ de dependencia ligera, y las que se encontraban en la $\geq 26$ semana reconocieron padecer dependencia moderada en un $33,3 \%$.
Tabla 5. Valoración funcional de las actividades sociales y recreativas

\begin{tabular}{|c|c|c|c|c|c|}
\hline \multirow[b]{2}{*}{ Variables } & \multirow{2}{*}{ Opc. } & \multicolumn{4}{|c|}{ Semanas de tratamiento } \\
\hline & & 5 sem & 8 sem & $15 \mathrm{sem}$ & $\geq 26$ sem \\
\hline Juegos De & $\mathrm{Si}$ & $1(20 \%)$ & $1(100 \%)$ & $2(40 \%)$ & $2(28,6 \%)$ \\
\hline Mesa & No & $4(80 \%)$ & $0(0 \%)$ & $3(60 \%)$ & $5(71,4 \%)$ \\
\hline \multirow{2}{*}{ Baile } & $\mathrm{Si}$ & $0(0 \%)$ & $1(100 \%)$ & $3(60 \%)$ & $2(28,6 \%)$ \\
\hline & No & $5(100 \%)$ & $0(0 \%)$ & $2(40 \%)$ & $5(71,4 \%)$ \\
\hline \multirow{2}{*}{ Manualidades } & $\mathrm{Si}$ & $4(80 \%)$ & $0(0 \%)$ & $1(20 \%)$ & $3(42,9 \%)$ \\
\hline & No & $1(20 \%)$ & $1(100 \%)$ & $4(80 \%)$ & $4(57,1 \%)$ \\
\hline $\begin{array}{l}\text { Ejercicios } \\
\text { dirigidos }\end{array}$ & $\mathrm{Si}$ & $5(100 \%)$ & $1(100 \%)$ & $5(100 \%)$ & $7(100 \%)$ \\
\hline $\begin{array}{l}\text { Ejercicios } \\
\text { libres }\end{array}$ & $\mathrm{Si}$ & $5(100 \%)$ & $1(100 \%)$ & $5(100 \%)$ & $7(100 \%)$ \\
\hline \multirow{2}{*}{ Deporte } & $\mathrm{Si}$ & $0(0 \%)$ & $0(0 \%)$ & $0(0 \%)$ & $0(0 \%)$ \\
\hline & No & $5(100 \%)$ & $1(100 \%)$ & $5(100 \%)$ & $7(100 \%)$ \\
\hline \multirow{2}{*}{ Caminatas } & $\mathrm{Si}$ & $5(100 \%)$ & $1(100 \%)$ & $5(100 \%)$ & $6(85,7 \%)$ \\
\hline & No & $0(0 \%)$ & $0(0 \%)$ & $0(0 \%)$ & $1(14,3 \%)$ \\
\hline \multirow{2}{*}{$\begin{array}{l}\text { Escuchar } \\
\text { radio }\end{array}$} & $\mathrm{Si}$ & $4(80 \%)$ & $1(100 \%)$ & $5(100 \%)$ & $6(85,7 \%)$ \\
\hline & No & $1(20 \%)$ & $0(0 \%)$ & $0(0 \%)$ & $1(14,3 \%)$ \\
\hline \multirow{2}{*}{ Ver TV } & $\mathrm{Si}$ & $5(100 \%)$ & $1(100 \%)$ & $5(100 \%)$ & $6(85,7 \%)$ \\
\hline & No & $0(0 \%)$ & $0(0 \%)$ & $0(0 \%)$ & $1(14,3 \%)$ \\
\hline \multirow{2}{*}{$\begin{array}{l}\text { Leer } \\
\text { periódicos }\end{array}$} & $\mathrm{Si}$ & $3(60 \%)$ & $1(100 \%)$ & $5(100 \%)$ & $3(42,9 \%)$ \\
\hline & No & $2(40 \%)$ & $0(0 \%)$ & $0(0 \%)$ & $4(57,1 \%)$ \\
\hline \multirow{2}{*}{ Leer Libros } & $\mathrm{Si}$ & $5(100 \%)$ & $1(100 \%)$ & $5(100 \%)$ & $4(57,1 \%)$ \\
\hline & No & $0(0 \%)$ & $0(0 \%)$ & $0(0 \%)$ & $3(42,9 \%)$ \\
\hline \multirow{2}{*}{$\begin{array}{l}\text { Reuniones } \\
\text { familiares }\end{array}$} & $\mathrm{Si}$ & $5(100 \%)$ & $1(100 \%)$ & $5(100 \%)$ & $5(71,4 \%)$ \\
\hline & No & $0(0 \%)$ & $0(0 \%)$ & $0(0 \%)$ & $2(28,6 \%)$ \\
\hline
\end{tabular}

Según la Tabla 5, en todas las semanas de tratamiento los sujetos afirmaron no practicar deporte (100\%). En la 5 semana de tratamiento, el $80 \%$ de los sujetos no practicaba juegos de mesa, y un $40 \%$ no realizaba lectura de periódicos. En la semana 8, el $100 \%$ no elaboraba manualidades, pero en la 15 semana de tratamiento un $80 \%$ no ejecutaba manualidades, y un $60 \%$ no participaba en juegos de mesa. En $\geq 26$ semana, el $71,4 \%$ no realizaba juegos de mesa, ni practican el baile; en tanto que $57,1 \%$ no realizaba manualidades ni leía periódicos

\section{Discusión}

Al comparar las investigaciones consultadas $(14,15)$ y la presente, los resultados obtenidos coinciden en que la actividad de mayor dependencia es la de subir y bajar escaleras. En este sentido, la encuesta de Salud, Bienestar y Envejecimiento (SABE) en América Latina y el Caribe, encontró que el 20\% de los adultos mayores que viven en la comunidad tenían limitación al menos en una AVD (16).

De acuerdo con Marzona, los pacientes con fibrilación auricular presentan mayor dependencia en la realización de las actividades de la vida diaria. Igualmente, este autor manifiesta limitaciones en las 
funciones ejecutivas, tales como realizar múltiples tareas y la secuencia en la realización de las tareas, lo que dificulta su desempeño en AIVD (17).

En este mismo sentido, Massucci expresa que la comorbilidad cardiovascular en pacientes con deficiencias neurológicas incrementa sus alteraciones funcionales (18).

En cuanto a las AIVD en los hombres, se puede notar que se presenta mayor dependencia en el manejo de asuntos económicos. Estos resultados coinciden con lo expuesto por otros autores quienes encontraron que la participación en la actividad económica de las personas mayores es escasa y que cuando aparece, no responde exactamente a una opción voluntaria, sino mas bien a la necesidad de garantizar un mínimo de recursos básicos para sobrevivir (17). Además, el ingreso por horas trabajadas es notoriamente inferior al recibido por las personas de 50 a 59 años.

La capacidad de las personas de disponer de bienes en general, económicos y no económicos, constituye un elemento clave en la calidad de vida en la vejez. Precisamente, la seguridad económica de las personas mayores se define como la capacidad de disponer y usar de forma independiente una cierta cantidad de recursos económicos regulares, así como de montos suficientes para asegurar una buena calidad de vida en la vejez (19).

Por otra parte, el goce de la seguridad económica permite a las personas mayores satisfacer las necesidades objetivas que propician una buena calidad de vida y les brindan independencia en la toma de decisiones. Además, esta seguridad mejora su autoestima, al permitirles el desempeño de roles significativos y la participación en la vida cotidiana como ciudadanos con plenos derechos (19).

Un adulto mayor dependiente que convive en el seno de una familia puede condicionar una serie de fenómenos en el funcionamiento de esta (19). Por ello, es importante disminuir las limitaciones funcionales que obstaculicen el desarrollo de actividades que el adulto mayor necesite y desee realizar, a través de intervenciones oportunas que retarden o detengan la aparición de la limitación en las actividades y de restricciones en la participación (9).

De las entrevistas realizadas a los cuidadores y/o familiares, se puede inferir que estos influyen en el desarrollo de algunas actividades de la vida diaria de los adultos mayores, lo cual puede fundamentarse en el temor a que el esfuerzo genere complicaciones en el estado actual de las personas mayores. Es necesario, entonces, seguir realizando investigaciones sobre cómo influyen los cuidadores y/o familiares en la dependencia de los adultos mayores.

Vale decir que, con base en los resultados, se diseño una guía de prevención de dependencia para los adultos mayores con enfermedad cardiovascular dirigida al familiar y/o persona responsable del cuidado de estos pacientes, con el fin de ayudar al paciente y a la familia a mantener e, incluso, mejorar los resultados positivos que obtuvieron en el funcionamiento de las AVD.

\section{Referencias}

1. Naciones Unidas. Seguimiento de la Segunda Asamblea Mundial sobre el Envejecimiento. 2009. http://www.un.org/esa/population/publications/W PA2009/WPA2009

2. Paz CJ. Vejez y políticas de envejecimiento en Latinoamérica y en Colombia: una realidad y un desafío. Rev. Aso. Colomb. Gerontol. Geriatr. 2011; 25 (2): 1525-1535

3. Ocampo CJ, Londoño AI. Ciclo vital individual: vejez. Rev. Asoc. Colomb. Gerontol. Geriatr. 2007; 21 (3): 1072-1084.

4. González A, Rangel C. Calidad de vida en el adulto mayor. Instituto de Geriatría. 365- 378. Disponible en: http://www.geriatria.salud.gob.mx/descargas/34.p df

5. Romero GJ, Mercedes R, González R. La cultura física como complemento de una buena salud física y mental en los Círculos de Abuelos. Acción. 2011; 7 (14): 28-31.

6. World Health Organization. The World Health report 2002:reducing risks, promoting healthy life. Geneva, Switzerland. WHO: 2002;1-230

7. Pan American Health Organization. Colombia Health Profile. In: PAHO. ed. Health in the Americas. II edition. Washington, D.C.: PAHO; 2003: 181-93

8. Magnus B, Pereira AC, Zuianello SR. Aptitud cardiorespiratoria y calidad de vida postinfarto en diferentes intensidades del ejercicio. Rev. Arq. Bras. Cardiol. 2010; 95 (3): 399-403. 
9. Ocampo CJ, Valencia PA, González RF. Envejecimiento y familia. Rev. Aso. Colomb. Gerontol. Geriatr. 2009; 23 (2):1259-1274.

10. Sanhueza PM, Castro SM, Merino J. Adultos mayores funcionales: un nuevo concepto en salud. Rev. Ciencia y Enfermería. 2005; 11(2): 17-21.

11. Acosta QC, González CR. Actividades de la vida diaria en adultos mayores: experiencia de dos grupos focales. Revista Psicología y salud. 2009; 19 (2): 289-293.

12. Espinosa BA, Romero CA. Evaluación geriátrica: metas, método e implementación. Rev. Aso. Colomb. Gerontol. Geriatr 2008; 22 (3): 11841191.

13. Montenegro SL, Salazar RC, De la Puente OC, Gómez SA, Ramírez AE. Aplicación de la evaluación geriátrica integral en los problemas sociales de adultos mayores. Acta Med Per. 2009; 26(3): 169-174

14. Rosas EG, Vélez AC. Fisioterapia y Rehabilitación Cardiaca, 1era ed. Universidad Autónoma de Manizales, 2009.

15. Graf SC. The Lawton instrumental activities of daily (IADL) Scale. MEDSURG Nursing. 2009;18:315-316

disponible en http://www.annalsoflongtermcare.com/article/745 3

16. Albala C, Lebrao ML, Leon DE, Ham-Chade R, Hennis AJ, Palloni A, et al. Encuesta salud, bienestar y enevejecimiento (SABE): metodología de la encuesta y perfil de la población estudiada. Rev Panam. Salud. Pública. 2005; 17 (5/6):307-22

17. Marzona I, O’Donnell M, Koon T, Craig A, Bosch J, Yusuf S. Increased risk of cognitive and functional decline in patients with atrial fibrillation: results of the ONTARGET and TRANSCEND studies. CMAJ. 2012; 184(6): 329-336.

18. Massucci M, Perrero L, Mantellini E. Petrozzino S, Gamma F, Nocella A, et al. Cardiorespiratory comorbidity: a new challenge for physical and rehabilitation medicine specialist. Eur $\mathbf{J}$ PhysRehabil Med. 2011; 48(1):1-8.

19. Dueñas E, Martínez M, Morales B, Muñoz C, Viafara S, Herrera J. Síndrome del cuidador de adultos mayores discapacitados y sus implicaciones Psicosociales. Revista Colomb Med. 2006; 37 (2) (Supl 1): 31-38.

Cienc. innov. salud. Junio 2014; 2 (1):33 - 39. Universidad Simón Bolívar (Col). ISSN: 2344-8636 http://portal.unisimonbolivar.edu.co:82/rdigital/innovacionsalud 